\title{
Efecto de la fisioterapia en un paciente con neurofibromatosis tipo II. Reporte de un caso
}

\author{
Effect of physiotherapy in a patient with neurofibromatosis type II. A case report \\ Julio César Granados Carrera 1,a; 2,3,4,ad, Javier Espinoza Castillo", ${ }^{1, \mathrm{~b}}$, Jimmy Alexander Mego Vásquez ${ }^{1, \mathrm{c}}$, \\ Eduardo Guzmán Huamán 1,b
}

\section{RESUMEN}

La fisioterapia en pacientes con lesión neurológica degenerativa puede mejorar la capacidad funcional permitiéndole una participación social activa y una mejor calidad de vida mientras la evolución de la enfermedad lo permita. Se describe los efectos de la fisioterapia en un paciente con lesión medular secundaria a Neurofibromatosis tipo II. La intervención se basó en terapias cardiorrespiratoria, funcional y deportiva. Se obtuvo en el paciente mayor funcionalidad para caminar, mejor autoestima y capacidad física para trabajar.

PALABRAS CLAVE: Fisioterapia, neurofibromatosis, traumatismos de la médula espinal. (Fuente: DeCS BIREME).

\section{SUMMARY}

Physiotherapy in patients with degenerative neurologic conditions may improve functional capacity allowing a better quality of life and active social participation, as long as the progression of disease allows it. We report the effects of physiotherapy in a patient with medullar involvement due to type II neurofibromatosis. Interventions were based on cardiorespiratory, functional and sport interventions. The intervention attained better functionality to walk, better self-confidence and physical capacity to work.

KEY WORDS: Physical therapy specialty, neurofibromatosis, spinal cord injuries. (Source: MeSH NLM).

\section{INTRODUCCIÓN}

La neurofibromatosis (NF), descrita por primera vez en 1882 por Von Recklinghausen, es una enfermedad genética caracterizada por una anormalidad neuro ectodérmica y por manifestaciones clínicas de afectación sistémica y progresiva que afectan principalmente a la piel, el sistema nervioso, los huesos, los ojos y otros órganos (1). Existen varios tipos, los principales son: El tipo I, la neurofibromatosis (NF-1), caracterizada por presentar manchas "café con leche" y crecimiento de dos o más neurofibromas (2); el tipo II (NF-2), se caracteriza por alta incidencia de tumores en el sistema nervioso (meningioma, ependimoma, neurofibroma espinal y glioma) asociadas a anomalías oculares, vestibulares y dérmicas (3); y el tipo III (NF3), o schwannomatosis caracterizada por desarrollar schwannomas múltiples, de evolución continua (4).

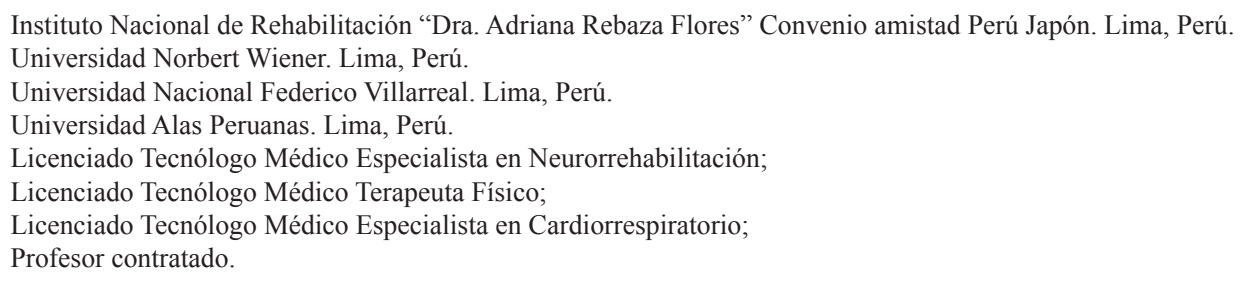


No hay tratamiento médico disponible para prevenir y revertir las lesiones. La atención se centra en asesoramiento genético, en la detección temprana, en el uso de fármacos y la resección quirúrgica de los tumores (1), y pocas veces fisioterapia y rehabilitación (5).

Se reporta el caso de un paciente con NF-2, de presentación inusual con lesión medular (LM), con múltiples complicaciones y con antecedente familiar de 2 hermanos fallecidos por la misma enfermedad, con el objetivo de describir los efectos de la fisioterapia cardiorrespiratoria, funcional y deportiva.

\section{PRESENTACIÓN DEL CASO}

Varón de 34 años, con diagnóstico clínico de NF-2, de 20 años de evolución. Inició síntomas hace 6 años de forma progresiva. Al examen clínico, se encontró debilidad en extremidades, hipoestesia en extremidades y tronco, dolor neuropático a nivel lumbar, alteraciones vestibulares y pérdida del equilibrio, alteraciones visuales, trastornos de la vejiga, intestino, sexual y psicológico que le provocó limitación para caminar, agarrar objetos, baja autoestima y restricción para trabajar.
Inició rehabilitación en el Departamento de lesiones medulares del Instituto Nacional de Rehabilitación (INR), siendo hospitalizado para tratamiento integral por 4 meses. El diagnóstico médico fue cuadriplejía espástica ASIA C nivel C4 de etiología no traumática, recibiendo tratamiento fisioterapéutico: cardiorrespiratorio, funcional y deportivo; además, evaluaciones médicas, terapia ocupacional, psicología, reeducación de la vejiga, intestino y orientación sexual (6). Cada sesión de fisioterapia tuvo en promedio 60 minutos a tolerancia, de lunes a sábados distribuidos en turnos mañana y tarde.

La fisioterapia cardiorrespiratoria consistió en mejorar la capacidad vital y la condición física mediante técnicas kinésicas de ventilación y el trabajo muscular de los brazos en la bicicleta ergonómica $(7,8)$; la fisioterapia funcional se aplicó para mejorar el alineamiento y la estabilidad del tronco en actividades anti gravitatorias mediante el trabajo muscular excéntrico y de relajación para mitigar el dolor (9); y como actividad deportiva practicó el tenis de mesa de pie para estimular el equilibrio y mejorar la autoestima (10). En cada intervención se evaluó el inicio y final para describir los cambios (Tablas 1,2,3 y 4).

Tabla 1. Evaluación de la función respiratoria.

\begin{tabular}{lcc}
\hline Evaluación & Inicial & Final \\
\hline Saturación de oxigeno & $97 \%$ & $97 \%$ \\
Distensibilidad torácica (cm) & 0,8 & 1,5 \\
Capacidad inspiratoria (ml) & 2,500 & 3,800 \\
Pico flujo espiratorio (lpm) & 250 & 400 \\
Tos & Débil No & Funcional \\
& funcional & asistida \\
\hline
\end{tabular}

Tabla 2. Evaluación de la función cardiaca al esfuerzo.

\begin{tabular}{|c|c|c|c|c|c|c|c|c|}
\hline & \multicolumn{2}{|c|}{ Distancia } & \multicolumn{2}{|c|}{ Tiempo } & \multicolumn{2}{|c|}{ Escala de Borg } & \multicolumn{2}{|c|}{$\begin{array}{l}\text { Tiempo de } \\
\text { recuperación }\end{array}$} \\
\hline & Inicio & Final & Inicio & Final & Inicio & Final & Inicio & Final \\
\hline Ergómetro de brazos & 0,5 millas & 3 millas & $10 \mathrm{~min}$ & $15 \mathrm{~min}$ & $\begin{array}{l}0 / 2 \\
2 / 5\end{array}$ & $\begin{array}{l}0 / 1 \\
1 / 3\end{array}$ & $10 \mathrm{~min}$ & $5 \mathrm{~min}$ \\
\hline $\begin{array}{l}\text { Test de caminata } 6 \\
\text { minutos }\end{array}$ & $450 \mathrm{~m}$ & $560 \mathrm{~m}$ & $6 \mathrm{~min}$ & $6 \min$ & $\begin{array}{l}0 / 2 \\
3 / 5\end{array}$ & $\begin{array}{l}0 / 1 \\
1 / 3\end{array}$ & $10 \mathrm{~min}$ & $5 \mathrm{~min}$ \\
\hline
\end{tabular}


Tabla 3. Evaluación del control postural parado.

\begin{tabular}{llll}
\hline Función & \multicolumn{1}{c}{ Evaluación } & \multicolumn{1}{c}{ Inicial } & \multicolumn{1}{c}{ Final } \\
\hline Postural & Cabeza y tronco & $\begin{array}{l}\text { Cabeza en extensión y rotación a la } \\
\text { derecha. } \\
\text { Tronco en flexión y rotación a la } \\
\text { izquierda }\end{array}$ & $\begin{array}{l}\text { Cabeza y tronco próximo a la } \\
\text { línea media. }\end{array}$ \\
& Pelvis, rodillas y tobillos & $\begin{array}{l}\text { Pelvis en retroversión } \\
\text { Rodillas y tobillos en valgo } \\
\text { moderado }\end{array}$ & $\begin{array}{l}\text { Pelvis neutra } \\
\text { Rodillas y tobillos en valgo leve }\end{array}$ \\
& $\begin{array}{l}\text { Dedos del pie y miembro } \\
\text { inferior }\end{array}$ & $\begin{array}{l}\text { Dedos flexionados, aducidos (en } \\
\text { garra) }\end{array}$ & Dedos alineados \\
Actividad & Marcha & Dependiente de andador agazapado & Camina sin andador y erguido \\
& Subir y bajar escaleras 1 piso & Dependiente & $\begin{array}{l}\text { Realiza cogiéndose de los } \\
\text { pasamanos } \\
\text { Realiza con supervisión }\end{array}$ \\
\hline
\end{tabular}

Tabla 4. Evaluación deportiva en tenis de mesa parado.

\begin{tabular}{llll}
\hline Función & Evaluación & Inicial & Final \\
\hline Gesto motor & Para golpear la pelota con la paleta & $\begin{array}{l}\text { Inestable, se coge de } \\
\text { mesa }\end{array}$ & $\begin{array}{l}\text { Estable sin cogerse de la } \\
\text { mesa }\end{array}$ \\
& $\begin{array}{l}\text { Desplazamientos laterales para } \\
\text { golpear la pelota } \\
\text { Rotación de tronco }\end{array}$ & $\begin{array}{l}\text { Perdida del equilibrio } \\
\text { Equilibrio lateral }\end{array}$ & Rógido \\
$\begin{array}{l}\text { Coordinación Parcial } \\
\text { velocidad }\end{array}$ & $\begin{array}{l}\mathrm{N}^{\circ} \text { de lanzamientos de la pelota } \\
\text { frente a la pared en 1 minuto sin } \\
\text { apoyo }\end{array}$ & 2 lanzamientos & 10 lanzamientos \\
& $\begin{array}{l}\mathrm{N}^{\circ} \text { de veces que lanza la pelota al } \\
\text { área contraria en 1 minuto sin apoyo }\end{array}$ & 1 lanzamiento & 25 lanzamientos \\
& $\begin{array}{l}\text { No de veces que golpea la pelota sin } \\
\text { perder el equilibrio en 1 minuto sin } \\
\text { apoyo }\end{array}$ & 2 veces & 18 veces \\
\hline
\end{tabular}

\section{DISCUSIÓN}

La fisioterapia busca la recuperación funcional en la disfunción del movimiento y toma importancia en las enfermedades neurodegenerativas (11). Los resultados describen que la fisioterapia provocó cambios al final de la rehabilitación; porque mejoró la capacidad vital y la condición física del paciente lo suficiente como para caminar y agarrar objetos y reforzó la autoeficacia de participar en el trabajo.
No se han encontrado estudios sobre el efecto simultáneo de estas tres modalidades de fisioterapia en la NF-2. Con respecto a la fisioterapia cardiorrespiratoria, Real (7), resalta que el programa de rehabilitación intensiva diaria contribuye a mejorar la capacidad cardiorrespiratoria en pacientes con enfermedades neurodegenerativas como la esclerosis múltiple, por extensión considerando que la NF-2 es una enfermedad degenerativa, la distensibilidad torácica, la capacidad inspiratoria y el pico flujo espiratorio mejoraron con la 
fisioterapia en forma progresiva pues tuvimos sesiones diarias y planificadas por el equipo de terapia física, lo que permitió un monitoreo adecuado de sus capacidades.

Con respecto a la terapia funcional, MaklesKacy (12), reportó que la fisioterapia mejoró de la funcionalidad física de la cara y aspectos psicológicos después de un trasplante quirúrgico facial y en un caso de NF-1, el paciente motivo de este reporte tuvo cambios positivos en el control postural y actividades de parado lo suficiente para caminar sin andador por tramos cortos y medios. Dericioğlu (9), publicó que la fisioterapia basada en el concepto Bobath en un niño con NF-1 mejoró la funcionalidad y postura a través del alineamiento y estabilidad, este resultado es similar al de nuestro paciente pues se aplicó el enfoque de este concepto, y el paciente obtuvo una mejor retroalimentación sensorial para mantener la postura.

Muraki (10), propone que el deporte en personas con lesión medular cuadripléjicas y parapléjicas puede mejorar la función física y la condición psicológica. Gonzáles-Cutre (13), describe que el deporte en un niño con NF-1 disminuyó los efectos negativos funcionales. En el manejo el paciente fue sometido a un entrenamiento deportivo de tenis de mesa en parado para reforzar su estabilidad lo que le permitió mejorar la tolerancia al esfuerzo físico mientras camina y manipula objetos funcionales, percibiendo un mejor balance vertical y mayor independencia.

En conclusión, la intervención paralela de la fisioterapia cardiorrespiratoria, funcional y deportiva tuvo un efecto positivo en la capacidad funcional y participación laboral en un paciente con NF-2 después de un periodo de 4 meses en rehabilitación integral. Sin embargo, somos conscientes que la evolución de esta discapacidad puede variar en cualquier momento y disminuir la funcionalidad obtenida.

\section{Agradecimientos:}

A la Jefatura del Departamento de Lesiones Medulares del Instituto Nacional de Rehabilitación y a la Directora de Docencia e Investigación por el apoyo brindado durante el proceso del estudio.

\section{Declaración de financiamiento y de conflictos de intereses:}

El estudio fue financiado por el Instituto Nacional de Rehabilitación. Los autores declaran no tener conflictos de intereses.

\section{Contribución de autoría:}

JCGC, JEC, JAMV y EGH, participaron en la recolección de los datos del caso, elaboración del reporte y en la discusión.

\section{Correspondencia:}

Julio César Granados Carrera

Av. A Mz. K Lote 29 Urb. San Antonio de

Carapongo. Lurigancho-Chosica

Lima15, Perú

Correo electrónico: jcgraca40@hotmail.com

Teléfono: 51979380871

\section{REFERENCIAS BIBLIOGRÁFICAS}

1. Antonio JR, Goloni-Bertollo EM, Trídico LA. Neurofibromatosis: chronological history and current issues. An Bras Dermatol. 2013; 88(3):329-43.

2. Duong T, Sbidian E, Valeyrie-Allanore L, et al. Mortality Associated with Neurofibromatosis 1: A Cohort Study of 1895 Patients in 1980-2006 in France. Orphanet Journal of Rare Diseases; 2018; 6(1):18-25.

3. Patel C, Ferner R, Grunfeld E. A qualitative study of the impact of living with neurofibromatosis type 2 . Psychology, Health \& Medicine. 2018; 16(1):19-28.

4. Plotkin SR, Blakeley JO, Evans DG, et al. Update From the 2011 International Schwannomatosis Workshop: From Genetics to Diagnostic Criteria. Am J Med Genet A. 2013; 161A(3):405-16.

5. Blakeley JO, Plotkin SR. Therapeutic advances for the tumors associated with neurofibromatosis type 1, type 2, and schwannomatosis. Neuro-Oncology. 2016; 18(5):624-638.

6. Harvey L. Tratamiento de la lesión medular. Guía para fisioterapeutas. Barcelona: Editorial Elsevier; 2010. p. 6-48.

7. Real Y, López M, Hernández, Díaz R, Cabrera J. Efectividad de un programa de rehabilitación respiratoria en pacientes con esclerosis múltiple. Revista Cubana de Salud Pública. 2010; 37(1):12-18.

8. Tørhaug T, Brurok B, Hoff J, Helgerud J, Leivseth G. Arm crank and wheelchair ergometry produce similar peak oxygen uptake but different work economy values in individuals with spinal cord injury. Biomed Res Int. 2016; 2016:5481843. doi: $10.1155 / 2016 / 5481843$

9. Dericioğlu B, Ilhan H. Effectiveness of physiotherapy and rehabilitation in type 1 neurofibromatosis; 18 months follow up. Pediatric Rehabilitation Congress Abstracts. Journal of Exercise Therapy and Rehabilitation. 2017; Sup (2): S78. Disponible en: http://www.jetr.org.tr/wp-content/uploads/pediatribildiri.pdf 
10. Muraki S, Tsunawake N, Hiramatsu S, Yamasaki M. The effect of frequency and mode of sports activity on the psychological status in tetraplegics and paraplegics. Spinal Cord. 2000; 38:309-314.

11. Smith R, Jones G, Curtis A, Murphy H, Flint G. Are established methods of physiotherapeutic management for long-term neurological conditions applicable to 'orphan' conditions such as syringomyelia? Physiotherapy Research International. 2016; 21(1): 4-21.
12. Makles-Kacy I, Zys-Owczarek E, Polak A, Maciejewski A. Physiotherapy in the first three months after allograft face transplantation - case report. Physiotherapy. 2016; 24(1):34-38.

13. González-Cutre D. Aplicación de un programa de actividad física para un niño con neurofibromatosis. Lecturas: Educación física y deportes. Oct 2005; 89. http://www.efdeportes.com/efd89/neurofib.htm

Recibido: $12 / 02 / 2018$

Aceptado: 26/03/2018 